\section{Technikfolgenabschätzung im Technikunterricht}

\author{
Vorstellung einer Diplomarbeit und ihrer \\ Ergebnisse
}

\author{
von Jean-Paul Löhr, Karlsruhe
}

Herstellung, Gebrauch und veränderte Verwendungszusammenhänge von Technik betreffen jeden Einzelnen und sind teilweise mit Folgen erheblicher Reichweite verbunden. Technisches Handeln muss folglich auch von jedem Einzelnen verantwortet werden. In diesem Beitrag wird der Frage nachgegangen, wie Technikfolgenabschätzung in der schulischen Bildung zu verankern ist. „TA im Unterricht“ wird hier als sachliche, kritische Auseinandersetzung mit technikbezogenen gesellschaftlichen Problemen beschrieben. Ziel von TA im Unterricht soll sein, einen Beitrag zu leisten, dass Schüler Verantwortung beim technischen Handeln übernehmen, sie zu mündigen Bürgern in einer technisierten Welt werden und Hilfestellungen zur geistigen und seelischen Bewältigung von Technik erfahren. ${ }^{1}$

\section{Definition von TA im Rahmen schulischer Bildung}

„Unter Technikfolgenabschätzung werden wissenschaftliche und kommunikative Beiträge zur Lösung technikbezogener gesellschaftlicher Probleme verstanden", so Armin Grunwald in seiner Einführung in die Technikfolgenabschätzung (Grunwald 2002, S. 52). Wie könnte auf dieser Grundlage eine TA, die im Rahmen schulischer und allgemeiner Bildung gelehrt wird, aussehen?

TA im Unterricht bedeutet inhaltlich die sachliche, kritische Auseinandersetzung mit technikbezogenen gesellschaftlichen Problemen.

Wissenschaftlichkeit wird in der Schule zwar angestrebt, ist aber im schulischen Alltagsbetrieb, v. a. bis einschließlich der Sekundarstufe I allenfalls ansatzweise umzusetzen. Im Unterricht einzulösen, ist jedoch eine Sachlichkeit, die eine wissenschaftsorientierte und ausgewogene Arbeitsweise erfordert. Diese Sachlichkeit würde auch die Chance der Distanzierung und somit der tiefgreifenden Reflexion einer Technik und sich selbst gegenüber beinhalten. Die „kritische Auseinandersetzung mit technikbezogenen gesellschaftlichen Problemen" beinhaltet in dieser Definition, dass gesellschaftliche Kontexte und die Rolle einer Technik darin offen hinterfragt werden sollen.

Mit dem Aspekt der technikbezogenen gesellschaftlichen Probleme wird verdeutlicht, dass die Technik weiterhin im Mittelpunkt des Interesses steht, aber im unauflöslichen Verbund mit gesellschaftlichen Fragen.

Zwar können in der Schule kaum Beiträge zur Lösung der Probleme beigesteuert werden. Zweck und Ziel einer TA im Unterricht sollte das vertiefte Nachdenken über Technik durch den Schüler in Bezug zu sich und der Welt sein (vgl. Tuchel 1967, S. 78; Wiesmüller 2009, S. 11). Demnach wird der Beitrag zur Lösung der technikbezogenen gesellschaftlichen Probleme darin liegen, einen eigenen reflektierten Standpunkt und eine eigene qualifizierte, nachvollziehbare Meinung zu einer bestimmten Thematik aufzubauen. Hier geht es um die Hinführung und Stärkung der Mündigkeit der Schüler.

Klaus Tuchel verdeutlicht in Bezug auf den Beitrag der Schule zu einer technischen Bildung im Sinne eines vertieften Nachdenkens über Technik durch den Schüler in Bezug zu sich und der Welt, dass er im ungebrochenen selbstverständlichen Gebrauch der Technik die Herausforderung sieht, die technische Entwicklung kritisch und distanziert zu durchdenken (vgl. Tuchel 1967, S. 78). Da für Klaus Tuchel Technik mehr als nur Konsum, nämlich auch Teil der eigenen Lebensgestaltung ist, sei ein kritisches Nachdenken über Technik und deren Folgen erforderlich: „In der Erziehung des jungen Menschen muss seine Nähe zur Technik aufgeschlossen und ergänzt werden durch die Schaffung einer Distanz zu ihr. [...] Kritische Distanz lässt sich aus dem Nachdenken sowohl über geschichtliche Herkunft als auch die gegenwärtige wirtschaftlich-politische Bedingtheit der Technik gewinnen, aus dem Durchschauen ihrer wissenschaftlichen Grundlage ebenso wie aus dem Durchdenken ihrer sozialen Folgen. Erst die in der Erziehung vorbereitete ErschlieBung von Nähe und Distanz zur Technik schafft die Möglichkeit ihrer vernünftigen Einbeziehung in unser Leben“" (Tuchel 1967, S. 78).

Christian Wiesmüller, der sich Gedanken zum Technikunterricht als Hilfe zur geistigen und 
seelischen Bewältigung von Technik gemacht hat, stellt die Frage, wie frei Menschen in der Technosphäre eigentlich sind (Wiesmüller 2009, S. 10). Damit fragt er nach den Möglichkeiten und Grenzen von Technik sowie nach der Problematik, wie Menschen die durch Technik gegebene Herausforderung geistig und psychisch bewältigen können. Welche Rolle werden Menschen in einer immer stärker technisierten Welt spielen?

Wiesmüller zeigt auf, dass Bildung eine lebenslange Anstrengung von Menschen sei, sich als freies geistiges Wesen zu entwickeln und so den Herausforderungen durch Technik zu begegnen. Denn Technik habe zwar zur Befreiung des Einzelnen beigetragen, aber sie führe in ihrer Ambivalenz auch zu einer kollektiven Abhängigkeit. Es sei demnach Aufgabe der Schule, die Bewältigung einer expandierenden Technik auf einer Ebene der nüchternen Handlungskompetenz wie auch auf der Ebene des Seelischen und Geistigen anzubahnen bzw. zu begleiten (Wiesmüller 2009, S. 10ff.). In Abschätzung zukünftiger Technologien ist nach Wiesmüller zu erwarten, dass jedem Einzelnen qualitativ mehr Verantwortlichkeit abverlangt werde als das rein instrumentelle Beherrschen von Technik, denn es könne nicht alles erlaubt sein, was (technisch) möglich oder gewollt wird. In diese neue Haltung der Verantwortlichkeit müsse aber grundlegend eingeführt werden.

\section{Didaktische Begründung konkretisiert am Beispiel „Service-Roboter“}

Robotik als Thema in der Schule bzw. im Technikunterricht erscheint gerade heute, da die Entwicklung und die konkrete Anwendung große Fortschritte macht und Roboter Einzug in unseren Alltag halten, notwendig. Die Technik muss verstanden werden, um ihr gewachsen zu sein und den Alltag (mit ihr) meistern zu können. Ein Verharren rein in der Sachperspektive bzw. die unzureichende Beachtung der Fachdidaktik durch die Techniklehrer scheint gängige Praxis im Technikunterricht zu sein. Ein gesellschaftlich so bedeutendes Thema wie die Robotik, die in naher Zukunft noch so manche Veränderung bewirken wird, wird in der Schule wohl oft nicht ausreichend aus einer Sinn- und Wertperspektive betrachtet und reflektiert (Schmayl 2010, S. 23; Löhr 2011, S. 53ff.). Schon in der Schule, dem Ort der institutionalisierten Hinführung zur Mündigkeit, zur verantwortungsvollen Eingliederung in die Gesellschaft hat eine Auseinandersetzung mit den Handlungen und der Technik sowie den daraus resultierenden Folgen stattzufinden (Schmayl 2010, S. 11f.; Sachs 2001, S. 6f.).

Was könnte eine TA zum Thema „ServiceRoboter" im Technikunterricht beinhalten? Die Etablierung der TA im Unterricht als ein die Problem- und Handlungsfelder übergreifender, fest verankerter Inhalt des Technikunterrichts im Sinne der Beschäftigung mit den Herausforderungen der Technik an ausgewählten Beispielen, wie z. B. „Service-Roboter in unterschiedlichen Lebensbereichen", könnte einen großen Gewinn darstellen.

Im Folgenden werden mögliche methodische Grundformen samt beispielhafter Themen und Beschreibungen zur Umsetzung von TA als Inhalt im Unterricht aufgelistet:

\section{(Historische) Technikstudie, auch als WebQuest}

Mit der Methode der Technikstudie nach den Definitionen von Winfried Schmayl und Daniel Bienia soll die Erschließung der sozial-humanen Zusammenhänge der Technik im Wechselspiel zwischen technischen Entwicklungen und gesellschaftlichen Veränderungen, bedingt durch Werte, Normen, Ideen, Meinungen und politische Entscheidungen, ermöglicht werden (Schmayl 1999, S. 14; Bienia 2004, S. 140ff.). Als WebQuests werden Lehr- und Lernarrangements bezeichnet, die über das Inter- oder Intranet zur Verfügung gestellt werden und die zum Ziel haben, Schülern Quellen als Ausgangspunkte für die eigenständige Bearbeitung einer Aufgabe zur Verfügung zu stellen, um eine selbstständige Konstruktion von Wissen zu ermöglichen (Landesakademie für Fortbildung und Personalentwicklung an Schulen in Baden-Württemberg 2012).

In einer Kombination dieser beiden Methoden ließen sich auch Themen wie „Roboter in der Landwirtschaft - Kein Problem?!“ und „Automaten und Roboter im Alltag von Gestern und Heute" im Unterricht erarbeiten: Auch außerhalb der Industrie haben sich Roboter etabliert. Die Informationen hierzu sind umfangreich und gut zugänglich. Mithilfe eines WebQuest wird der Suchraum für die Schüler eingegrenzt und er- 
leichtert. Fragen- oder aufgabengestützt können die Schüler Studien zu diesem Thema anfertigen, d. h. den „State of the Art" beschreiben, erweiterte Fragen stellen und Stellung beziehen.

\section{Fallstudie}

Eine Fallstudie ist nach den Beschreibungen von Fritz Wilkening und Winfried Schmayl eine praxisbezogene, fachübergreifende Methode zum Erlernen einer mündigen Problemlösung und Entscheidungsfindung in komplexen Zusammenhängen (Schmayl 1999, S. 14; Wilkening 1977, S. 123ff.).

In einer Fallstudie, beispielsweise unter dem Titel „Können Roboter Pflegepersonal im Bereich der Altenpflege ersetzen?“, könnten die Schüler anhand von Fragen nach den technischen Möglichkeiten, dem gesellschaftlichen Bedarf, den Anliegen von Pflegebedürftigen oder der medizinischen/fürsorglichen Notwendigkeit zu einer Beurteilung der Lage und einer daran anschließenden Entscheidung kommen.

\section{Technolog}

Christian Wiesmüller beschreibt diese Methode in Anlehnung an ein Logbuch oder einen Blog. Dieses subjektive Selbstgespräch in schriftlicher Form könne nach ihm auf das Oberthema „Ich und die Technik" konzentriert werden. Technik und ihre Folgen würden zu einem persönlichen und geäußerten Thema aus subjektiver Sicht (Wiesmüller 2009, S. 17).

Unter der Überschrift „Meine Welt MIT oder OHNE Roboter" könnten sich Schüler mit folgenden Fragen beschäftigen, Statements dazu abgeben und sich v. a. mit dieser Thematik selbstreflexiv auseinandersetzen: Wo begegne ich Robotern in meinem Alltag? Inwieweit bin ich schon auf Roboter angewiesen? Wie ist meine Beziehung zu Robotern? Wie könnte sich meine Beziehung zu Robotern ändern? Was erhoffe ich mir von Robotern der Zukunft? Welche Befürchtungen habe ich in Bezug auf Roboter?

\section{Kleines Technikmuseum}

Auch diese methodische Überlegung wurde von Christian Wiesmüller beschrieben. Die Grundin- tention ist die Umsetzung des musealen Gedankens des Sammelns, Ausstellens und der kritischen Auseinandersetzung mit Artefakten durch die Schüler (Wiesmüller 2009, S. 286).

Die Schüler könnten sich unter den Themen „Bildnisse von Menschen und Robotern“ bzw. „Manuelle Arbeit - Automaten - Roboter" mit Robotern und Vorstellungen bzw. Beschreibungen von diesen befassen oder sie könnten sich mit selbstkonstruierten Modellen den Werdegang von Automaten und Robotern aufzeigen und ihre Ergebnisse ausstellen. Im Sinne einer TA sollte ein besonderes Augenmerk auf die Auswirkungen der Etablierung von Robotern auf die Gesellschaft gelegt werden: Arbeitsmarkt, Wohlstand, Gesundheit, Freizeit, Gefühlslagen und Stimmungen.

In Anlehnung an ein Analyseinstrument der Politikwissenschaft und die „Kategorien politischer Bildung in der Phasenstruktur einer Problemanalyse" nach Sutor wird in Tabelle 1 ein Raster zur Strukturierung von TA-Themen für den Unterricht vorgestellt (Sutor 1992; Meyer 2000, S. 83ff.).

Nach Auswahl eines Themas in der Vorphase schließen sich drei Phasen an: (1) die Situationsanalyse mit der Frage, was ist, (2) die Möglichkeitserörterung mit der Frage, was sein wird, und (3) die Phase der vorläufigen Urteilsbildung mit der Frage, was geschehen sollte. Alle drei Phasen sind durch die drei Dimensionen - Form/ Struktur, Inhalt und Prozess - gleichermaßen durchdrungen. Beispielhaft soll dies in Tabelle 2 veranschaulicht werden.

So soll es möglich sein, die Bedingungen und Folgen möglichst umfassend zu erfassen und die Thematik zu durchdringen, entscheidende Fragen zu stellen und ein vorläufiges Urteil zu fällen. Des Weiteren dient es der Vorbereitung des Themas durch den Lehrer, kann aber auch eigesetzt werden, um den Schüler bei der Bearbeitung eines TA-Themas im Unterricht behilflich zu sein.

\section{Anmerkung}

1) Der Artikel basiert auf den Ergebnissen meiner Diplomarbeit zum Thema „Technikfolgenabschätzung im Technikunterricht", die ich im WS 2010/2011 an der Pädagogischen Hochschule Karlsruhe bei Prof. Wolf Bienhaus und Prof. Dr. Christian Wiesmüller abgeschlossen habe. 
Tab. 1: Raster zur Strukturierung von TA-Themen im Technikunterricht

\begin{tabular}{|c|c|c|c|}
\hline \multicolumn{4}{|l|}{ Raster } \\
\hline \multirow[t]{3}{*}{ Vorphase } & \multirow{2}{*}{\multicolumn{3}{|c|}{$\begin{array}{l}\text { Auswahl und Planung des Themas nach: } \\
\text { Aktualität/Betroffenheit/Interesse/Bedeutung/Zugänglichkeit }\end{array}$}} \\
\hline & & & \\
\hline & Form/Struktur & Inhalt & Prozess \\
\hline Was ist? & Vorhandene Technologien und & Ideen, Träume, Utopie & \\
\hline SITUATIONSANALYSE & Verfahren & Innovationen & Interessen \\
\hline Was wird sein? & Gesetze & Trends & Akteure \\
\hline MÖGLICHKEITSERÖRTERUNG & Normen & Forschungs- und Entwicklungsvorhaben & Betroffene \\
\hline Was sollte geschehen? & Werte & Bedarfe & Konflikt \\
\hline URTEILSBILDUNG & Gesellschaftliche Bedingungen & Problematiken & \\
\hline
\end{tabular}

Quelle: Eigene Darstellung

Tab. 2: Exemplarische Veranschaulichung zum Umgang mit dem Raster zur Strukturierung von TAThemen im Technikunterricht am Beispiel von Servicerobotern in der Pflege

\begin{tabular}{|c|c|c|}
\hline \multicolumn{3}{|c|}{ Beispiel: Fragen zum TA-Thema Roboter in der Pflege } \\
\hline & Mögliche Fragen & Beispielhafte Kurzantworten \\
\hline \multirow{3}{*}{ Situationsanalyse } & $\begin{array}{l}\text { Form/Struktur: } \\
\text { Welche Roboter für den Einsatz in der Pflege } \\
\text { existieren schon? }\end{array}$ & $\begin{array}{l}\text { Care-O-bot, } \\
\text { Paro }\end{array}$ \\
\hline & $\begin{array}{l}\text { Inhalt: } \\
\text { Welche Ziele werden derzeit bei der Entwick- } \\
\text { lung von Robotern für die Pflege verfolgt? }\end{array}$ & $\begin{array}{l}\text { Sichere und autonome Handreichungen; } \\
\text { Unterhaltung; Fortbewegung }\end{array}$ \\
\hline & $\begin{array}{l}\text { Prozess: } \\
\text { Wer ist daran interessiert, Roboter in der Pfle- } \\
\text { ge einzusetzen? }\end{array}$ & $\begin{array}{l}\text { Gesellschaft - demografischer Wandel; } \\
\text { Pfleger - Entlastung bei der Arbeit; } \\
\text { Pflegebedürftige - Wahrung der Intimsphäre; } \\
\text { Forschung }\end{array}$ \\
\hline \multirow{3}{*}{$\begin{array}{l}\text { Möglichkeitserörte- } \\
\text { rung }\end{array}$} & $\begin{array}{l}\text { Form/Struktur } \\
\text { Welche Werte werden durch den Roboterein- } \\
\text { satz tangiert? }\end{array}$ & $\begin{array}{l}\text { Persönlichkeitsrechte; } \\
\text { Privatsphäre; } \\
\text { Freiheit; } \\
\text { Datensicherheit }\end{array}$ \\
\hline & $\begin{array}{l}\text { Inhalt: } \\
\text { Welche Entwicklungen im Bereich der Robo- } \\
\text { tik werden von besonderer Relevanz sein? }\end{array}$ & $\begin{array}{l}\text { Selbstreferenzielle, lernende Roboter, hinsicht- } \\
\text { lich Autonomie, Sicherheit, Verantwortung und } \\
\text { Menschenbild }\end{array}$ \\
\hline & $\begin{array}{l}\text { Prozess: } \\
\text { Welche Folgen sind für die Betroffenen in } \\
\text { der Pflege durch den Einsatz von Robotern zu } \\
\text { erwarten? }\end{array}$ & $\begin{array}{l}\text { Stärkere Abhängigkeit von Maschinen; } \\
\text { Entwöhnung von Menschen }\end{array}$ \\
\hline \multirow{3}{*}{ Urteilsbildung } & $\begin{array}{l}\text { Form/Struktur: } \\
\text { Welche Gesetze könnten notwendig sein, den } \\
\text { Einsatz von Robotern in der Pflege zu regeln? }\end{array}$ & $\begin{array}{l}\text { Regelungen zu Einsatzzwecken und Einsatzzei- } \\
\text { ten von Robotern und Pflegern bei der Betreuung } \\
\text { der Bedürftigen }\end{array}$ \\
\hline & $\begin{array}{l}\text { Inhalt: } \\
\text { Welche Bedarfe sollten Roboter unbedingt } \\
\text { erfüllen? }\end{array}$ & $\begin{array}{l}\text { Menschenwürdiger Umgang mit Patienten; } \\
\text { sichere Betreuung von Demenzkranken }\end{array}$ \\
\hline & $\begin{array}{l}\text { Prozess: } \\
\text { Wie sind die Akteure hinsichtlich der Übernah- } \\
\text { me von Verantwortung einzubinden? }\end{array}$ & $\begin{array}{l}\text { Ethikkommissionen; } \\
\text { geteilte Verantwortung aller }\end{array}$ \\
\hline
\end{tabular}

Quelle: Eigene Darstellung 


\section{Literatur}

Beecroft, R.; Dusseldorp, M., 2009: TA als Bildung. Ansatzpunkte für Methodologie und Lehre. In: Technikfolgenabschätzung - Theorie und Praxis 18/3 (2009), Karlsruhe, S. 55-64; http://www.itas.fzk.de/ tatup/093/bedu09a.pdf (download 11.1.12)

Berken, D., 2010: Technikfolgenabschätzung als Gegenstand technischer Allgemeinbildung. In: tu - Zeitschrift für Technik im Unterricht 138 (2010), Villingen-Schwenningen, S. 12-20

Bienia, D., 2004: Technikgeschichte als Gegenstand allgemeiner technischer Bildung. Didaktische und methodische Aspekte für den Technikunterricht. Hamburg Grunwald, A., 2002: Technikfolgenabschätzung Eine Einführung. Berlin

Landesakademie für Fortbildung und Personalentwicklung an Schulen in Baden-Württemberg, 2012: Was sind WebQuests? http://lehrerfortbildung-bw.de/unterricht/ webquest/definition.html (download 16.4.12)

Löhr, J.P., 2011: Technikfolgenabschätzung im Technikunterricht. Diplomarbeit PH Karlsruhe

Meyer, T., 2000: Was ist Politik? Opladen

Sachs, B., 2001: Technikunterricht: Bedingungen und Perspektiven. In: tu - Zeitschrift für Technik im Unterricht 100/2 (2001), Villingen-Schwenningen, S. 5-12

Schmayl, W., 2010: Didaktik allgemeinbildenden Unterrichts. Baltmannsweiler

Schmayl, W., 1999: Zur Methodik des Technikunterrichts. In: tu - Zeitschrift für Technik im Unterricht 93/3 (1999), Villingen-Schwenningen, S. 5-15

Sutor, B., 1992: Politische Bildung als Praxis: Grundzüge eines didaktischen Konzepts. Schwalbach

Tuchel, K., 1967: Herausforderung der Technik. Gesellschaftliche Voraussetzungen und Wirkungen der technischen Entwicklung. Bremen

Wiesmüller, C., 2009: Technikunterricht als Hilfe zur geistigen und seelischen Bewältigung der Technik. In: tu - Zeitschrift für Technik im Unterricht 131 (2009), Villingen-Schwenningen, S. 10-17

Wilkening, F., 1977: Unterrichtsverfahren im Lernbereich Arbeit und Technik. Ravensburg

\section{Kontakt}

Jean-Paul Löhr

Rhode-Island-Allee 49, 76149 Karlsruhe

E-Mail: loehrs@googlemail.com

\section{Stadtverkehr der Zukunft Was denken junge Karlsruherinnen und Karlsruher?}

\section{von Maike Puhe und Jens Schippl, ITAS}

\section{Projekthintergrund}

Auf dem Weg zur Arbeit oder zur Schule, in der Freizeit oder für alltägliche Erledigungen - Menschen sind darauf angewiesen, mobil zu sein. Schneller, zuverlässiger, sicherer, kostengünstiger und möglichst umweltverträglicher Verkehr ist ein zentrales Ziel nachhaltiger Stadtplanung, trägt maßgeblich zu einer hohen städtischen Lebensqualität bei und leistet einen wesentlichen Beitrag zur wirtschaftlichen Prosperität einer Stadt. Das stetige Verkehrswachstum, insbesondere des motorisierten Individualverkehrs, führt hingegen zu einer reduzierten Effizienz des Verkehrssystems und beeinträchtigt die Lebens- und Umweltqualität der Städte. Neben steigenden $\mathrm{CO}_{2}$-Emissionen sind Menschen in städtischen Agglomerationen den Folgen von Lärm, Stau, Unfallrisiko und Flächenverbrauch ausgesetzt. Für die Zukunft wird besonders beim Güterverkehr weiteres Wachstum erwartet, wenn beim Personenverkehr eine gewisse Sättigung eintritt (infas, DLR 2010).

Die Entwicklung und Verbreitung innovativer Technologien und Nutzungskonzepte spielt in vielen Konzepten zur Lösung der genannten Herausforderungen in verschiedenen urbanen Räumen eine wichtige Rolle. Neue Antriebstechnologien wie die Elektromobilität, aber auch Informations- und Kommunikationstechnologien sollen helfen, Verkehr sauberer zu machen, auf umweltfreundlichere Verkehrsmittel zu verlagern oder gar zu vermeiden und so negative Auswirkungen wie z. B. $\mathrm{CO}_{2}$-Emissionen zu reduzieren. ${ }^{1}$

Das STOA-Projekt „Urban Transport“, das den inhaltlichen Rahmen dieses Beitrags liefert, betrachtet Innovationen, die den oben genannten Herausforderungen entgegenwirken, sowie Faktoren, welche die Diffusion dieser Innovationen beeinflussen können. Ziel des Projekts ist es, Erfolg versprechende Entwicklungspfade für zukünftiges politisches Vorgehen aufzuzeigen, welche zu einem nachhaltigen städtischen Verkehrssystem 